\title{
PRIMATE METACARPAL MORPHOLOGY AND DETERMINATION OF THUMB OPPOSABILITY AND POTENTIAL TOOLMAKING FROM FOSSIL HOMINIDS.
}

REGA*, Elizabeth A. Department of Biology, Cal State University, San Bernardino, CA 92407 USA; PARKER, Greig, Dept. Of Archaeology and prehistory, University of Sheffield, Sheffield, England.

In recent years, a number of comparative studies have focused on the morphology of the hominoid hand, specifically features thought to reflect the differing manual capabilities of humans and apes. Contrast can be drawn between the "truly opposable" thumb of humans, where pad to pad contact involving substantial 1st metacarpal movement is made, with more limited "pseudo-opposability" of ape thumbs, with phangeal hyperflexion and little 1st metacarpal movement. Despite an extremely fragmentary human fossil record, many have argued that "human-like" hands appear at the same time as the earliest stone tools.

Recent work by Susman $(1988,1991)$ proposes that hand bones classified as Paranthropus robustus demonstrate true thumb opposability, and links ability to manufacture stone tools with thumb opposability. He furthers the connection (1994) with the hypothesis that stone tool-making capabilities can be identified in the fossil record by the metrical and morphological affinities of the 1st metacarpal.

In this paper, we test this hypothesis and whether such differences can be linked unambiguously to stone tool manufacture in Paranthropus. As part of a larger ongoing study of pollical bones, we present results of the analysis the 1st metacarpals of Homo sapiens sapiens, Gorilla gorilla, Pan troglodytes, Pongo pygmaeus, Papio hamadryas, Papio doguera, Papio anubis, Colobus guerezcand Cercopithecus mitis. The specimens, with exception of the human are all housed at the British Museum of Natural History. The human specimens are from medieval cemetery collections housed at the University of Bradford. The interpretation of these data will be discussed with reference to a range of factors, including sex, age, lateralisation (handedness), body weight, locomotory behaviour, and tool productionluse, all of which may influence hand morphology. Measurement of the 1st metacarpal were taken following the methodology of Susman (1994). Femoral and humeral dimensions were measured to enable body weight estimates to be made from published regression equations for comparison. Muscle attachment sites of the Palmar Interosseous, the Dorsal Interosseous, the Opponens Pollicis, and the Abductor Pollicis Longus were graded on ordinal scale. All four muscle attachment sites were plotted to test for interspecific and left right side differences.

The results of this study demonstrate there while there are differences in the mean size and shape of the metacarpal 1, for humans, gorillas, chimpanzees and orang-utans, there is considerable overlap in $\mathrm{MC1}$ index. Human, gorilla and chimpanzee all show a large overlap in the relationship of length to articular head width. These species also show a considerable overlap in the strength of their muscle This is opposed to Susman's $(1994 ; 1995)$ claims. Chimpanzees and humans show similar body weight to MC1 Index ratios, and show a small overlap at their higher and lower ranges respectively. Gorillas and the Old World Monkeys show a much different relationship. The sample size is small and the values are quite variable, but these species do not show the clear association between body weight and MC1 Index that is present in humans and chimpanzees. The data from this study suggest that body weight is an important factor in the width of the articular head. Thus, although broad patterns are clear from the data, the overlap between hominoids of different species renders inference from the limited and fragmentary fossil record tenuous.

SUSMAN, R.L. 1988. Hand of Paranthropus robustus from Member I Swartkrans: fossil evidence for tool behaviour. Science 240:781-784.

SUSMAN, R.L. 1991. Who made the Oldowan tools? Fossil evidence for tool behaviour in PlioPleistocene hominids. Journal of Anthropological Research 47:129-151.

SUSMAN, R.L. 1994. Fossil evidence for early hominids. Science 265:1570-1573.

SUSMAN, R.L. 1995. Thumbs, tools and early hominids. Science 268: 589. 